\title{
Emission of Submillimeter Electromagnetic Waves by Coherent Phonons
}

\author{
T. Dekorsy, H. Auer, C. Waschke, H. J. Bakker, H. G. Roskos, and H. Kurz \\ Institut für Halbleitertechnik II, Rheinisch-Westfälische Technische Hochschule Aachen, D-52056 Aachen, Germany \\ V. Wagner and P. Grosse \\ I. Physikalisches Institut, Rheinisch-Westfälische Technische Hochschule Aachen, D-52056 Aachen, Germany
}

\begin{abstract}
We report on the first observation of the emission of electromagnetic radiation from coherent lattice vibrations in a semiconductor. Coherent optical phonons are excited by ultrashort laser pulses in singlecrystal tellurium. THz frequency radiation emitted by the Dember-field-driven phonons is detected by time-resolved THz-emission spectroscopy. The measurements are complemented by optical pump-probe experiments with a polarization-sensitive detection scheme utilizing the symmetry of the Raman tensor.
\end{abstract}

The investigation of coherent phonons in solid-state materials excited with ultrashort laser pulses is a subject of great current interest. In recent years, coherent phonons have been observed in the transient optical reflectivity and in the transmission of several semiconductors $[1-3]$. For a certain class of materials including Te, only coherent optical phonons of $A_{1}$ symmetry have been detected in femtosecond time-resolved experiments [2], although modes of different symmetry have been identified in cw-infrared (IR) reflectivity and Raman scattering experiments. The selective excitation of $A_{1}$ modes has been explained by a displacive excitation mechanism, in which a strong interband excitation leads to an ultrafast displacement of the atomic equilibrium position $[2,4]$. We present a detailed study of coherent phonons in Te. In addition to the $A_{1}$ mode, phonons of $E$ symmetry are observed in time-resolved measurements of the anisotropic reflectivity changes (RC), obeying selection rules given by the symmetry of the Raman tensor. Since all phonons in Te of symmetry different from $A_{1}$ are IR active, their coherent excitation is expected to be accompanied by the emission of electromagnetic radiation. In this Letter, we report on the first time-resolved observation of $\mathrm{THz}$ emission that originates from the macroscopic polarization associated with coherent longitudinal optical (LO) phonons. The radiation is detected with $\mathrm{THz}$-emission spectroscopy that allows the direct measurement of the amplitude and phase of the coherent electromagnetic radiation emitted after pulsed optical excitation [5].

The IR activity of phonons in the single-element semiconductor Te arises from the strong electronic polarizability and the lack of inversion symmetry [6]. Te crystallizes in a hexagonal lattice (space group $D_{3}^{4}$ or $D_{3}^{6}$ ) consisting of three atoms per unit cell arranged in a helix along the $c$ axis. The lattice vibrations consist of a fully symmetrical, only Raman-active $A_{1}$ mode $(3.6 \mathrm{THz})$, two degenerate Raman- and IR-active $E$ modes $\left(E_{\mathrm{TO} / \mathrm{LO}}^{\prime}: 2.76 / 3.09 \mathrm{THz}\right.$, $E_{\mathrm{TO} / \mathrm{LO}}^{\prime \prime}: 4.22 / 4.26 \mathrm{THz}$ ), and one only IR-active $A_{2}$ mode $\left(A_{2, \mathrm{TO} / \mathrm{LO}}: 2.6 / 2.82 \mathrm{THz}\right)[7]$. The internal polarization is either perpendicular ( $E$ modes) or parallel ( $A_{2}$ mode) to the $c$ axis. All experiments are performed on two different Te single crystals with the $c$ axis perpendicular $(c \perp)$ and parallel $(c \|)$ to the surface.

To prove the excitation of coherent phonons different from the $A_{1}$ mode, we perform optical pump-probe measurements and detect the anisotropic RC. The laser source is an unamplified colliding-pulse mode-locked (CPM) laser delivering pulses with $2 \mathrm{eV}$ photon energy, $50 \mathrm{fs}$ pulse duration, and $20 \mathrm{pJ}$ energy per pulse. The reflected probe beam is split with a polarizing beam splitter into two orthogonally polarized components $R_{j}$ and $R_{k}$, where $j$ and $k$ denote the orientation of the polarization in the Cartesian system of the crystal. The intensity difference $\Delta R_{j}-\Delta R_{k}$ is recorded as a function of time delay [1]. To enable a high-resolution determination of the phonon frequencies, we also measure the time derivative of the RC [8].

The manifestation of phonon modes in the anisotropic RC results from the modulation of the dielectric susceptibility by the coherent lattice vibration with amplitude $Q$. A perturbative expansion in $Q$ and truncation after the first term allows us to express the anisotropic RC as follows: $\Delta R_{j}-\Delta R_{k} \sim \vec{E}_{j}^{r}(\partial \chi / \partial Q) \vec{E}^{i} Q-\vec{E}_{k}^{r}(\partial \chi / \partial Q) \vec{E}^{i} Q$, where $\vec{E}^{i}$ and $\vec{E}^{r}$ are the incident and reflected probe fields, respectively. $(\partial \chi / \partial Q)_{j k}$ denotes the Raman tensor, which contains the following nonzero elements [7]:

$$
\begin{gathered}
E(x):(\partial \chi / \partial Q)_{x x}=-(\partial \chi / \partial Q)_{y y}=c ; \quad(\partial \chi / \partial Q)_{y z}=(\partial \chi / \partial Q)_{z y}=d, \\
E(y):(\partial \chi / \partial Q)_{x y}=(\partial \chi / \partial Q)_{y x}=-c ; \quad(\partial \chi / \partial Q)_{x z}=(\partial \chi / \partial Q)_{z x}=-d .
\end{gathered}
$$


The Raman tensor of the $A_{1}$ mode contains diagonal elements only. For the excitation of the $c \perp$ surface, we obtain for the anisotropic RC induced by the degenerate $E$ modes: $\Delta R_{x}-\Delta R_{y} \sim \sqrt{2} c\left(Q_{E(x)}+Q_{E(y)}\right)$. For the fully symmetric $A_{1}$ mode, $\Delta R_{x}-\Delta R_{y}$ is zero.

Figure 1(a) depicts the measured isotropic RC $\Delta R$ and the anisotropic RC $\Delta R_{x}-\Delta R_{y}$ of the $c \perp$ surface normalized to the isotropic reflectivity $R_{0}$ at an excitation density of $2 \times 10^{18}$ electron-hole pairs per $\mathrm{cm}^{3}$ [9]. The isotropic RC is strongly modulated by the coherent excitation of the $A_{1}$ mode [4]. The anisotropic RC is nearly 2 orders of magnitude smaller and has a complicated oscillatory structure. Figure 1(b) shows the Fourier transforms of the time-domain data recorded with the time-derivative technique. The frequency spectrum of the isotropic RC reveals a sharp peak at $3.60 \mathrm{THz}$ corresponding to the frequency of the Raman-active $A_{1}$ mode. Other modes are not resolved [10]. The Fourier transform of the anisotropic RC clearly reveals the two $E_{\mathrm{TO}}$ modes at $2.77 \mathrm{THz}$ and $4.2 \mathrm{THz}$. The fully symmetric $A_{1}$ mode is suppressed in the anisotropic RC, in accordance with the symmetry of the Raman tensor as discussed above. The $E_{\mathrm{LO}}$ modes are not observed, since the electro-optic and deformation potential contributions to the Raman signal cancel each other at this surface [11].

In Fig. 2, the Fourier transform of the oscillatory contribution of the anisotropic RC, $\Delta R_{x z}-\Delta R_{x,-z}$, of the $c \|$ surface is shown. In addition to the $E_{\mathrm{TO}}^{\prime}$ mode,
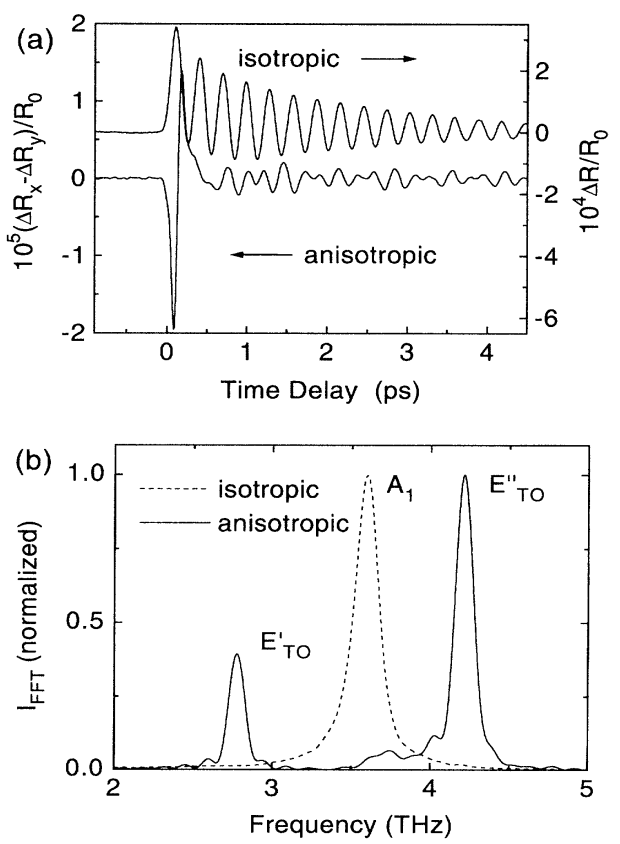

FIG. 1. (a) Isotropic ( $\Delta R$, upper curve) and anisotropic $\left(\Delta R_{x}-\Delta R_{y}\right.$, lower curve) reflectivity changes of $\mathrm{Te}, c \perp$ surface. (b) Fourier transforms of the experimentally recorded time derivatives of the reflectivity changes.

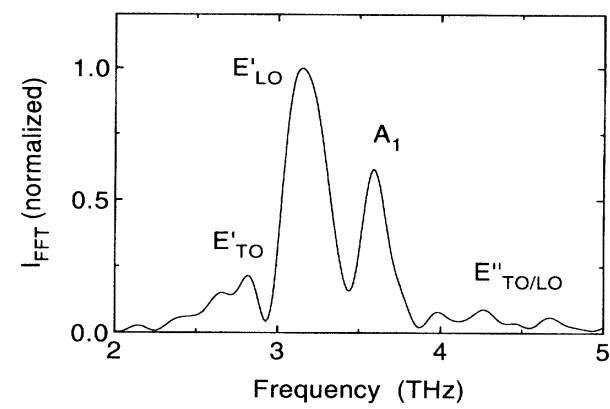

FIG. 2. Fourier transform of the oscillatory part of the anisotropic reflectivity change $\Delta R_{x z}-\Delta R_{x,-z}$ at the $c \|$ surface.

the $E_{\mathrm{LO}}^{\prime}$ mode appears in the Fourier spectrum of the anisotropic RC, while again the isotropic RC is dominated by the $A_{1}$ mode (not shown). The $A_{1}$ mode is not fully suppressed in the anisotropic RC because of residual surface scattering. At the $E_{\mathrm{TO} / \mathrm{LO}}^{\prime \prime}$ frequencies, only a weak signal is observed. The observation of the $E_{\mathrm{LO}}^{\prime}$ mode at this surface is in accordance with cw-Raman experiments [11]. The dephasing time of the $E_{\mathrm{LO}}^{\prime}$ is significantly shorter $(\sim 2 \mathrm{ps})$ than the dephasing time of the $E_{\text {TO }}^{\prime}$ phonon $(\sim 10 \mathrm{ps})$ due to the plasmon-phonon coupling of the longitudinal phonon mode with the optically excited carrier plasma [3].

Summarizing the pump-probe experiments, we have shown that all Raman-active phonons can be coherently excited and detected, while the only IR-active $A_{2}$ mode is not observed. The selection rules for the detection are determined by the symmetry of the Raman tensor.

In the THz-emission experiments, the sample is excited under $45^{\circ}$ incidence by Ti:Sapphire laser pulses with $1.75 \mathrm{eV}$ photon energy and a pulse duration of $150 \mathrm{fs}$ [12]. The coherent $\mathrm{THz}$ radiation emitted in the direction of the reflected optical beam is collected with two paraboloidal mirrors and detected with a submillimeter dipole antenna that is gated by a second time-delayed laser pulse. The current in the dipole antenna, which is proportional to the incident electric field transient, is recorded as a function of the time delay [5]. The sensitivity of the detection system peaks at $1 \mathrm{THz}$ and gradually rolls off to a cutoff at $4 \mathrm{THz}$ [5]. To investigate the polarization of the $\mathrm{THz}$ radiation, a wire-grid polarizer is placed before the antenna. The polarization sensitivity of the antenna itself is complex because of the birefringence of the sapphire substrate.

Figure 3(a) shows the measured electric field emitted from the $c \perp$ surface (time delay zero is chosen arbitrarily). The signal consists of a strong initial emission followed by a periodic modulation that can be traced over several picoseconds. The strong peak is attributed to a polarization that results from the ultrafast buildup of a strong photo-Dember field [13], which is driven by the strong carrier gradient at the surface and the difference 

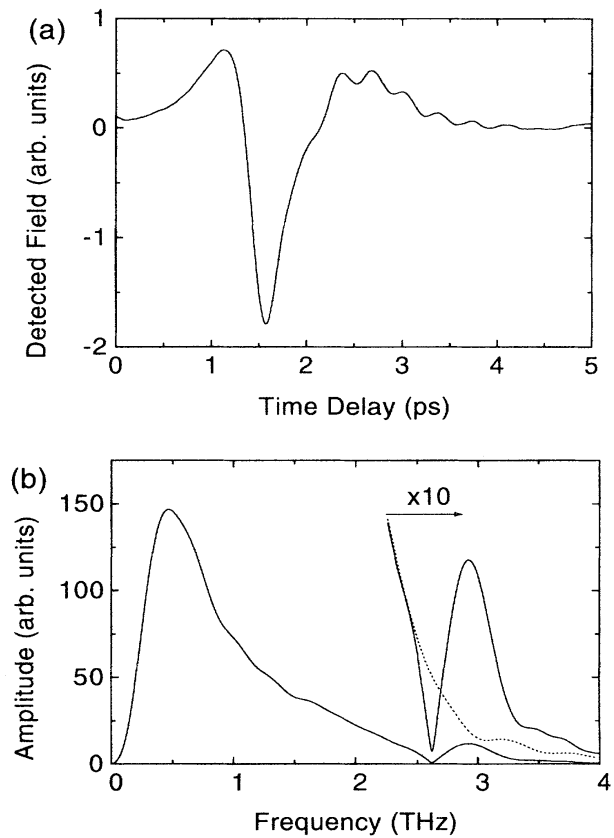

FIG. 3 (a) Detected electric field emitted from the $c \perp$ surface of Te. (b) Amplitude of the Fourier transform of the timedomain signal. The high frequency part is depicted also enlarged by a factor of 10 . The dashed line gives the high frequency part of the antenna response determined from the emission of a broadband emitter (InP, also $\times 10)$.

in electron and hole diffusion coefficients [14]. This effect is amplified by differences in the transient electron and hole temperatures. The screening of depletion-layer fields as the source of the radiation [5] can be neglected because the charged surface states have a low density and the band gap is small $(0.33 \mathrm{eV})[6]$. The amplitude of the initial $\mathrm{THz}$ signal due to the Dember field is only a factor of 5 weaker than the emission from a polar semiconductor with strong surface fields (e.g., InP) for the same excitation power. Figure 3(b) depicts the amplitude of the Fourier transform of the time-domain signal. The frequency spectrum reveals a broad peak at approximately $500 \mathrm{GHz}$. For high frequencies, the signal decreases to nearly zero at $2.62 \mathrm{THz}$ before reaching a second maximum at about $2.9 \mathrm{THz}$. For clarity, this part of the spectrum is enlarged by a factor of 10 . The high-frequency spectrum of a broadband emitting surface (InP, also $\times 10$, not normalized) is shown for comparison, representing the antenna response. The emission peak at $500 \mathrm{GHz}$ results from the carrier transport dynamics leading to the buildup of the Dember field. The high-frequency features correspond to the oscillatory part of the time-domain signal. A strong deviation of the $\mathrm{THz}$ emission from Te compared to the broadband emitter is clearly observed in this frequency range.

The fact that the frequencies of two IR-active phonon modes $\left(A_{2}\right.$ and $\left.E^{\prime}\right)$ overlap with the frequency range of interest, indicates that coherent phonons are the source of the oscillatory emission. Since the $A_{2}$ mode has an internal polarization perpendicular to the excited surface, while the $E^{\prime}$ mode is polarized parallel to the surface, a polarization-resolved analysis of the $\mathrm{THz}$ radiation allows the identification of the contributing modes.

Figure 4 depicts the THz signals emitted from the $c \perp$ surface with and without a wire-grid polarizer. The shift in the time delay results from the polarizer placed into the beam path of the $\mathrm{THz}$ radiation. The shape of the $p$ polarized $\mathrm{THz}$ radiation is similar to the radiation detected without polarizer. The oscillatory contribution remains clearly visible. The $p$-polarized emission originates from polarization changes perpendicular to the excited surface [5], i.e., the buildup of the longitudinal Dember field. In the $s$-polarized component, no contribution at phonon frequencies is observed and the signal shape has changed. The signal in this component must result from a different nonlinear polarization. Hence, the high-frequency oscillations in the $\mathrm{THz}$ emission result from an internal polarization perpendicular to the emitting surface, i.e., parallel to the $c$ axis.

From these measurements we conclude that the $A_{2}$ mode dominates the high-frequency $\mathrm{THz}$ spectrum of the $c \perp$ surface. The $E_{\mathrm{TO}}^{\prime}$ does not contribute, although it dominates the anisotropic RC in this frequency range. Thus we identify the dip at $2.6 \mathrm{THz}$ in the spectrum of Fig. 3(b) with the $A_{2, \text { TO }}$ mode, while the peak at $2.9 \mathrm{THz}$ results from the $A_{2, \mathrm{LO}}$ mode being a strong source of radiation.

The emission of radiation from coherent phonons with polarization perpendicular to the excited surface is explained as follows: Coherent LO phonons are excited by the ultrafast buildup of a Dember electric field directed perpendicular to the excited surface. In contrast to the LO phonon generation via the ultrafast screening of surface fields in GaAs [1,8], Te LO phonons are driven by the buildup of a Dember field. The coherent LO phonons are associated with a macroscopic longitudinal polarization at the LO frequency emitting dipole radiation, which is detected in the far field by the antenna. The resonant

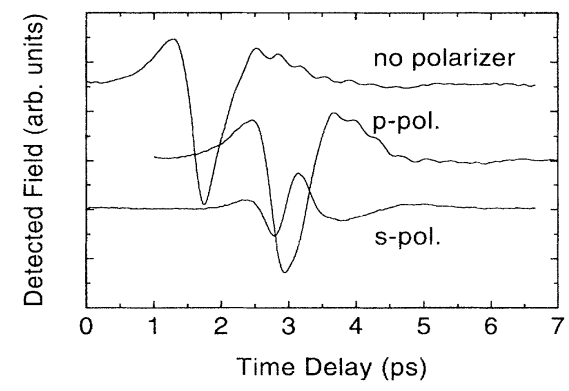

FIG. 4. THz field emitted from the $c \perp$ surface without polarizer and with polarizer placed before the antenna in $p$ and $s$ configuration. For clarity, the curves are shifted vertically. 
coupling of the longitudinal Dember field with the LO phonon gives rise to an amplification of the field at the LO frequency and a strong reduction of the field at the TO frequency. Such effects have also been predicted for the case of $\mathrm{THz}$ emission by coherent LO phonons in GaAs [15]. We observe emission at the phonon frequencies and not at other frequencies from plasmon-phonon coupled modes [15], although the average plasmon frequency is close to the optical phonon frequencies. We explain the absence of plasmon-phonon coupled modes from the strong inhomogeneity of the carrier density resulting from the extremely short absorption length.

The above interpretation of $\mathrm{THz}$ emission by phonons is verified by excitation of the $c \|$ surface (not shown). The Fourier spectrum of the $\mathrm{THz}$ emission looks qualitatively the same as for the $c \perp$ surface. Now a dip in the spectrum is observed at $2.7 \mathrm{THz}$ and a peak at $3.1 \mathrm{THz}$, which are close to the $E_{\mathrm{TO}}^{\prime}$ and the $E_{\mathrm{LO}}^{\prime}$ mode, respectively. In this case, the internal polarization of the observed phonon mode is again oriented perpendicular to the excited surface. At this surface, the Raman- and IRactive $E_{\mathrm{LO}}^{\prime}$ is observed in both, the $\mathrm{THz}$ emission and the anisotropic RC. The $E^{\prime \prime}$ mode is not observed, as it is above the frequency detection limit and has only a weakly polar character.

Finally, we discuss the relevance of (i) propagation and (ii) out-coupling effects of the $\mathrm{THz}$ radiation. The buildup of the Dember field driving the coherent LO phonons is itself associated with the broadband emission of radiation. (i) The propagation of the transverse electromagnetic radiation at frequencies close to an opticalphonon resonance can also lead to a spectral modulation of the $\mathrm{THz}$ radiation [16]. This propagation effect can be neglected, since the thickness of the emitting region of approximately $30 \mathrm{~nm}$ is much shorter than the absorption length of the $\mathrm{THz}$ radiation, which is larger than $1 \mu \mathrm{m}$ at the $E_{\mathrm{TO}}^{\prime}$ frequency. (ii) The out coupling of the $\mathrm{THz}$ radiation from the crystal into air is influenced by the strong wavelength dependence of the refractive index at the phonon resonance. Assuming equal radiation intensities at the LO and TO phonon inside the crystal, impedance matching leads to a higher radiation intensity at the LO than at the TO frequency outside the crystal. However, calculations show that this effect by itself cannot explain the high electric field amplitude at the LO frequency and the total absence of radiation at the TO frequency observed in our experiments.

In conclusion, we report on the coherent excitation of optical phonons in single-crystal tellurium. All Ramanactive phonon modes are observed in either the isotropic or the anisotropic time-resolved reflectivity changes in agreement with the symmetry of the Raman tensor. For the first time, we apply time-resolved $\mathrm{THz}$ emission spectroscopy for the investigation of coherent IR-active phonons. The $\mathrm{THz}$ emission by phonons of $A_{2}$ and $E$ symmetry is observed. The observation of the emission of light from coherent phonons opens a new field in the investigation of lattice dynamics.

We gratefully acknowledge G.C. Cho and A.V. Kuznetsov for helpful discussions. This work was supported by the Alfried-Krupp Stiftung and the Deutsche Forschungsgemeinschaft.

[1] G. C. Cho, W. Kütt, and H. Kurz, Phys. Rev. Lett. 65, 764 (1990).

[2] T. K. Cheng et al., Appl. Phys. Lett. 57, 1004 (1990); T. K. Cheng et al., Appl. Phys. Lett. 59, 1923 (1991).

[3] W. A. Kütt, W. Albrecht, and H, Kurz, IEEE J. Quantum. Electron. QE-28, 2434 (1992).

[4] H. J. Zeiger et al., Phys. Rev. B 45, 768 (1992).

[5] X.-C. Zhang and D.H. Auston, J. Appl. Phys. 71, 326 (1992); H. G. Roskos, Lith. J. Phys. 34, 175 (1994).

[6] P. Grosse, M. Lutz, and W. Richter, Solid State Commun. 5, 99 (1967); P. Grosse, The Physics of Tellurium, Springer Tracts in Modern Physics Vol. 48 (Springer, Berlin, 1969).

[7] P. Grosse and W. Richter, in Landolt-Börnstein, Numerical Data and Functional Relationships in Science and Technology, edited by O. Madelung, Landolt-Bornstein, New Series, Vol. 17 (Springer, Berlin, 1983).

[8] T. Pfeifer et al., Appl. Phys. A 55, 482 (1992).

[9] For the anisotropic reflectivity measurement, only the polarizing beam splitter and a second photo diode are placed into the path of the reflected probe beam, leaving the excitation conditions unchanged.

[10] $E$ modes with a scattering intensity only a factor of 5 smaller than that of the $A_{1}$ mode are observed in Raman spectra from the same sample. This difference between cw-Raman and time-resolved coherent-phonon experiments is a consequence of the displacive excitation mechanism (Refs. [2,4]).

[11] W. Richter, J. Phys. Chem. Solids 33, 2123 (1972).

[12] A Ti:Sapphire laser instead of the CPM is used in the $\mathrm{THz}$-emission experiments because of its much higher average power. The laser fluence at the sample is approximately the same for both experiments. The pumpprobe experiments are also performed with a Ti:Sapphire laser at $1.7 \mathrm{eV}$ energy, but the phonon amplitudes are higher for excitation with the CPM laser due to a broad resonance of the Raman process around $2.2 \mathrm{eV}$ (Ref. [11]).

[13] H. Dember, Phys. Z. 32, 554 (1931).

[14] The absorption length at $1.75 \mathrm{eV}$ is approximately $35 \mathrm{~nm}$ [6], resulting in a large carrier gradient along the sample normal. Model calculation predicts the buildup of electric fields as large as $50 \mathrm{kV} / \mathrm{cm}$ immediately after excitation. The relevance of the Dember effect is supported by intensity-dependent measurements to be published elsewhere.

[15] A. V. Kuznetsov and C. J. Stanton (private communication).

[16] M. Born and K. Huang, Dynamical Theory of Crystal Lattices (Oxford University Press, Oxford, 1954). 